\title{
PERIOD VARIATIONS IN THE BETA CEP STAR $\alpha$ LUP
}

\author{
E. CHAPELLIER and J.C. VALTIER \\ Observatoire de la Côte d'Azur, department Fresnel, B.P. 229, 06304 Nice cedex 4
}

$\alpha$ Lup (HR 5469) is a multiperiodic beta Cep star. According to Shobbrook (1979) and Lampers \& Goossens (1982) the main period ( $\mathrm{P}=0.25985$ day) corresponding to the radial mode is constant. The amplitude of the secondary period, attributed to a non radial mode, is so small that it does not affect the determination of maximum dates.

In order to determine the phase lag between light and radial velocity, we compared the photometric observations from Van Hoof, analysed by Lampens \& Goossens (1982) with the radial velocity measurements from Rodgers \& Bell (1962). We obtained a phase lag of 0.0579 day (0.22 period).

The $\mathrm{O}-\mathrm{C}$ diagram shows clearly a period increase of $0.28 \mathrm{sec}$. around the year 1974 .

The observations can be fitted by two ephemeris:

$1955-1974: \mathrm{Ml}=2435000.0082+0.25984595 \mathrm{E}$

$1974-1982: \mathrm{Ml}=2442000.2593+0.25984916 \mathrm{E}$

In some beta Cep stars, period changes have been explained by a binarity effect. Although $\alpha$ Lup is not considered as a binary, some variations of the gamma axis exist. In 1955 the value of the $\gamma$-axis measured on 80 lines by Pagel (1956) was $4 \mathrm{~km} / \mathrm{s}$ while the 9 lines measured by Mathias et al (1993) in 1989 ranged from 5.3 to $9.5 \mathrm{~km} / \mathrm{s}$ with a mean value of $7.25 \mathrm{~km} / \mathrm{s}$. Such a $3.25 \mathrm{~km} / \mathrm{s}$ difference would involve a $0.25 \mathrm{sec}$. increase of the pulsational period which is consistent with the observed variation.

$\alpha$ Lup is only 200 parsecs away, so it should be interesting to check by interferometry the existence of a companion. If the binary hypothesis is the good one, the orbital period should be greater than 50 years with large eccentricity.

\section{References}

Breger, M. : 1967, Monthly Notices of the RAS 136, 51

Heynderickx, D. : 1992, Astronomy and Astrophysics 96, 207

Hutchings J.B., Hill G. : 1980, Astronomy and Astrophysics, Supplement Series 42, 135

Lampens, P., Goossens M.: 1982, Astronomy and Astrophysics 115, 413

Lesh, J.R. : 1978, Astrophysical Journal 219, 947

Mathias, P., Aerts, C., De Pauw, M., Gillet, D., Waelkens, C.: preprint

Pagel, B.E.J. : 1956, Monthly Notices of the RAS 116, 10

Rodgers, A.W., Bell, R.A.: 1962, Observatory 82, 26

Shobbrook, R.R. : 1979, Monthly Notices of the RAS 189, 571 


\begin{tabular}{lrrl} 
DATE & Nights & O - C & References \\
\hline 35297.2722 & 14 & 0.0002 & Pagel (1956) \\
37511.9398 & 1 & 0.0008 & Rodgers \& Bell (1962) \\
38931.2155 & 3 & -0.0021 & Breger (1967) \\
41713.1290 & 1 & 0.0007 & Lesh (1978) \\
42882.4400 & 2 & 0.0049 & Hutchings \& Hill (1980) \\
43602.2294 & 13 & 0.0210 & Shobbrook (1979) \\
47224.7865 & 12 & 0.0657 & Heyndericks (1992) \\
\hline
\end{tabular}

$$
\text { O-C diagram of } \alpha \text { Lup }
$$

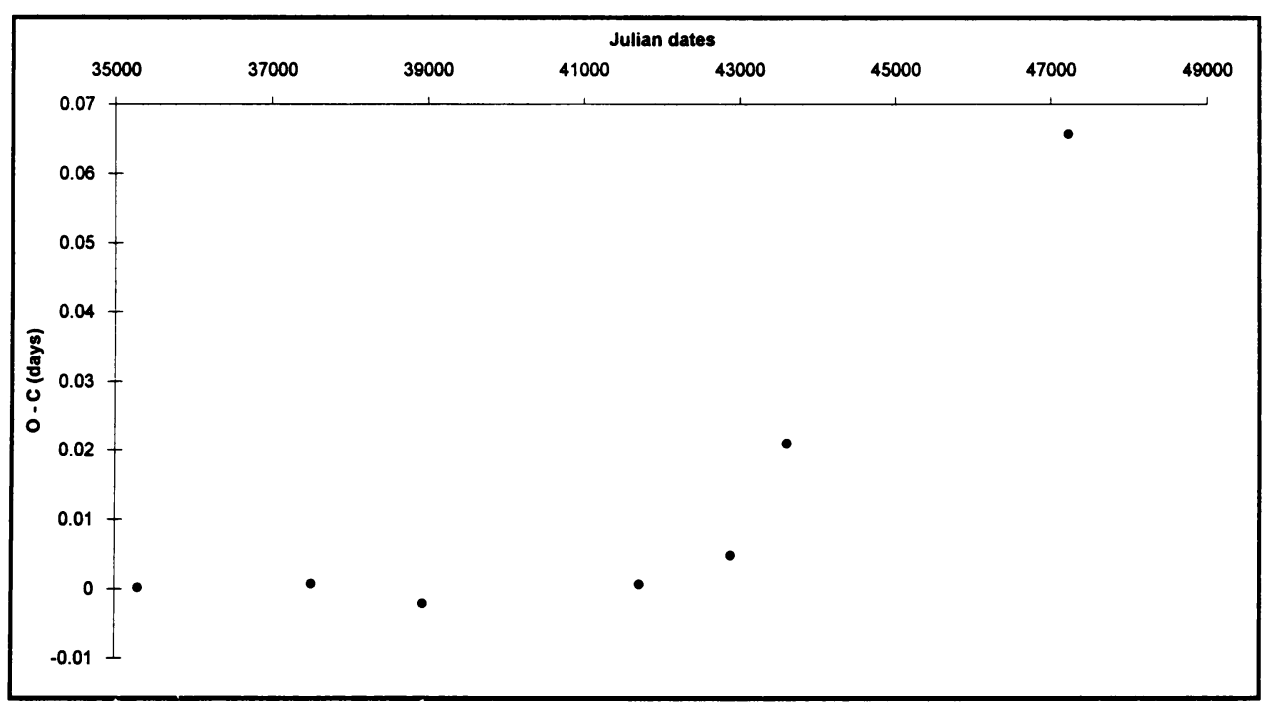

